QUARTERLY OF APPLIED MATHEMATICS

VOLUME LXIX, NUMBER 1

MARCH 2011, PAGES 79-89

S $0033-569 X(2010) 01231-3$

Article electronically published on December 9, 2010

\title{
NUMERICAL SOLUTION OF A SINGULAR INTEGRAL EQUATION WITH CAUCHY KERNEL IN THE PLANE CONTACT PROBLEM
}

\author{
BY \\ M. R. CAPOBIANCO (Istituto per le Applicazioni del Calcolo "Mauro Picone" - CNR, Sezione di \\ Napoli, Via Pietro Castellino 111, 80131 Napoli, Italy) \\ AND \\ G. CRISCUOLO (Dipartimento di Matematica e Applicazioni, Università degli Studi di Napoli \\ "Federico II", Complesso Monte Sant'Angelo, Edificio T, Via Cintia, 80126 Napoli, Italy)
}

Abstract. This paper describes a collocation method for solving numerically a singular integral equation with Cauchy and Volterra operators, associated with a proper constraint condition. The numerical method is based on the transformation of the given integral problem into a hypersingular integral equation and then applying a collocation method to solve the latter equation. Convergence of the resulting method is then discussed, and optimal convergence rates for the collocation and discrete collocation methods are given in suitable weighted Sobolev spaces. Numerical examples are solved using the proposed numerical technique.

1. Introduction. Let us consider the singular integral equation

$$
-\frac{1}{\pi} \int_{-1}^{1} \frac{v(x)}{x-t} d x+g(t) \int_{-1}^{t} v(x) d x+\frac{1}{\pi} \int_{-1}^{1} k(t, x) v(x) d x=f(t), \quad|t|<1,
$$

with the condition

$$
\int_{-1}^{1} v(x) d x=C
$$

where $C$ is a preassigned constant. Here the symbol $f_{-1}^{1}$ denotes the Cauchy principal value, and $g, f$ and $k$ are given functions on $[-1,1]$ and $[-1,1]^{2}$, respectively. Substituting $v=\sigma \bar{v}, \sigma^{-1}(x)=\varphi(x)=\sqrt{1-x^{2}}$ in (1.1), we shall consider in the sequel the equation written in operator form

$$
\left(-S_{\sigma}+M_{g} V_{\sigma}+K_{\sigma}\right) \bar{v}=f
$$

Received July 15, 2009.

2010 Mathematics Subject Classification. Primary 65R20; Secondary 41A05.

E-mail address: mariarosaria.capobianco@cnr.it

E-mail address: giuliana.criscuolo@unina.it 
where $\left(M_{g} h\right)(t)=g(t) h(t), S_{\sigma}$ and $V_{\sigma}$ are the Cauchy singular integral operator and the Volterra operator defined by

$$
\begin{gathered}
\left(S_{\sigma} \bar{v}\right)(t)=\frac{1}{\pi} \int_{-1}^{1} \frac{\bar{v}(x)}{x-t} \sigma(x) d x \\
\left(V_{\sigma} \bar{v}\right)(t)=\int_{-1}^{t} \bar{v}(x) \sigma(x) d x
\end{gathered}
$$

respectively, and $K_{\sigma}$ denotes the integral operator

$$
\left(K_{\sigma} \bar{v}\right)(t)=\frac{1}{\pi} \int_{-1}^{1} k(t, x) \bar{v}(x) \sigma(x) d x
$$

corresponding to the perturbation kernel $k$.

In practice equations such as (1.1) with (1.2) occur quite often in the theory of elasticity and fluid mechanics, in particular in the mathematical solution of the plane contact problem. A.I. Kalandija [9, Chapter Three] describes a series of problems which can be formulated in terms of equations such as (1.1) with a condition similar to (1.2). Applications of integral equations of the first kind with both Cauchy and Volterra operators are also related by G.M.L. Gladwell in [7. An exhaustive review of a collection of singular integro-differential equations arising from practical applications in applied mathematics and mathematical physics has recently been presented in 5 .

The collocation method for the above equation to be considered here, based on Chebyshev polynomials, represents the endpoint singularities of the exact solution. Lots of papers have dealt with the classical collocation or quadrature method for (1.3) dealing with the case $g \equiv 0$. Among others we refer the reader to [1] and the references cited therein. The aim of the present paper is to prove optimal convergence rates for collocation and quadrature methods for (1.3) in a suitable weighted Sobolev norm.

The paper is organized as follows: in Section 2, we give some notation and investigate the property of the integral equation (1.3); in Section 3, we present a procedure to solve (1.3) numerically, proving its stability and optimal convergence rates. Finally, in Section 4 , we report some numerical results and we give some computational considerations.

2. Notation and preliminaries. In order to make the paper more readable we start with some notation and preliminary results.

Let $L_{w}^{2}, w(x)=(1-x)^{\alpha}(1+x)^{\beta}, \alpha, \beta>-1$ denote the weighted space of square integrable functions on the interval $[-1,1]$ endowed with the scalar product and the norm

$$
\langle u, v\rangle_{w}=\frac{1}{\pi} \int_{-1}^{1} u(x) \overline{v(x)} w(x) d x, \quad\|u\|_{w}=\sqrt{\langle u, u\rangle_{w}}
$$

respectively. Moreover, let $p_{n}^{w}$ refer to the normalized Jacobi polynomial (with positive leading coefficient) of degree $n$ with respect to the Jacobi weight $w$. For real numbers $s \geq 0$ define the weighted Sobolev space $L_{w}^{2, s}$ by

$$
L_{w}^{2, s}=\left\{u \in L_{w}^{2}: \sum_{n=0}^{\infty}(1+n)^{2 s}\left|\left\langle u, p_{n}^{w}\right\rangle_{w}\right|^{2}<\infty\right\},
$$


with the norm

$$
\|u\|_{w, s}=\left[\sum_{n=0}^{\infty}(1+n)^{2 s}\left|\left\langle u, p_{n}^{w}\right\rangle_{w}\right|^{2}\right]^{1 / 2} .
$$

Let $T_{j}$ and $U_{j}$ be the Chebyshev polynomials of the first and of the second kind, respectively, normalized by $\left\langle T_{j}, T_{j}\right\rangle_{\sigma}=\pi / 2, j \neq 0,\left\langle T_{0}, T_{0}\right\rangle_{\sigma}=\pi$, and $\left\langle U_{j}, U_{j}\right\rangle_{\varphi}=\pi / 2$. Then, we define the spaces $H_{\varphi}^{2, s}$ and $\tilde{H}_{\sigma}^{2, s}$ to be the closures of all polynomials with respect to the norms \|\|$_{H_{\varphi}^{2, s}}=\|\|_{\varphi, s}$ and $\|u\|_{\tilde{H}_{\sigma}^{2, s}}=\left[\sum_{j=1}^{\infty} j^{2 s}\left|\left\langle u, T_{j}\right\rangle_{\sigma}\right|^{2}+\left|\left\langle u, T_{0}\right\rangle_{\sigma}\right|^{2}\right]^{1 / 2}$, respectively. For the operator $\bar{V}_{\sigma}=\sigma V_{\sigma}$, the following result holds.

THEOREM 2.1. The operator $\bar{V}_{\sigma}: \tilde{H}_{\sigma}^{2, s} \backslash\left\{T_{0}\right\} \longrightarrow H_{\varphi}^{2, s+1}$ is continuous with continuous inverse.

Proof. Let $\bar{V}_{\sigma} \mu=\nu$ and $\mu \in \tilde{H}_{\sigma}^{2, s} \backslash\left\{T_{0}\right\}$. Due to

$$
\bar{V}_{\sigma} T_{j}=-\frac{U_{j-1}}{j}, \quad j=1,2, \ldots,
$$

we have, with $\mu=\frac{2}{\pi} \sum_{j=1}^{\infty}\left\langle\mu, T_{j}\right\rangle_{\sigma} T_{j}$,

$$
\bar{V}_{\sigma} \mu=-\frac{2}{\pi} \sum_{j=1}^{\infty}\left\langle\mu, T_{j}\right\rangle_{\sigma} \frac{U_{j-1}}{j}
$$

yielding

$$
\left\langle\nu, U_{j}\right\rangle_{\varphi}=-\frac{\left\langle\mu, T_{j+1}\right\rangle_{\sigma}}{j+1}, \quad j=0,1, \ldots
$$

Hence,

$$
\|\nu\|_{H_{\varphi}^{2, s+1}}^{2}=\sum_{j=0}^{\infty}(j+1)^{2 s}\left|\left\langle\mu, T_{j+1}\right\rangle_{\sigma}\right|^{2}=\|\mu\|_{\tilde{H}_{\sigma}^{2, s}}^{2}-\left\langle\mu, T_{0}\right\rangle_{\sigma}^{2},
$$

from which the assertion follows.

In order to describe an algorithm for the numerical solution of the contact reactions represented by the singular integral equation (1.3), we start by observing that in view of the previous theorem the solution of the integro-differential problem (1.1)-(1.2) is equivalent to solving the hypersingular integral equation

$$
\left(A_{\varphi}+\hat{S}_{\varphi}+\hat{K}_{\varphi}\right) u=h
$$

where

$$
\begin{gathered}
u(t)=\sigma(t)\left[\int_{-1}^{t} \bar{v}(x) \sigma(x) d x-\frac{C}{2}(1+t)\right], \quad u(-1) \varphi(-1)=u(1) \varphi(1)=0, \\
h(t)=f(t)-\frac{C}{2}\left[(1+t) g(t)-\frac{1}{\pi} \log \frac{1-t}{1+t}+\frac{1}{\pi} \int_{-1}^{1} k(t, x) d x\right],
\end{gathered}
$$

and $A_{\varphi}, \hat{S}_{\varphi}, K_{\varphi}$ are the operators

$$
\begin{gathered}
\left(A_{\varphi} u\right)(t)=g(t) \varphi(t) u(t) \\
\left(\hat{S}_{\varphi} u\right)(t)=-\frac{1}{\pi} \frac{d}{d t} \int_{-1}^{1} \frac{u(x)}{x-t} \varphi(x) d x
\end{gathered}
$$




$$
\left(\hat{K}_{\varphi} u\right)(t)=-\frac{1}{\pi} \int_{-1}^{1} k_{x}(t, x) u(x) \varphi(x) d x .
$$

We remark that relation (2.4) respects the symmetry of the considered functions. In fact, if $\bar{v}$ is even, it follows that $u$ is odd. We use (2.3)-(2.4) as the basis for the proposed numerical method to solve the problem (1.1)-(1.2). This implies that an approximation of the solution $u$ is generated, but the desired approximation for the original solution $v$ does not require the additional step corresponding to the numerical differentiation (cf. relation (3.7)). Further, this reformulation allows us to use standard mathematical techniques (see [2, 3]) for the stability and the convergence analysis.

In the last few years, several authors have been working on the development of numerical methods for hypersingular integral equations similar to (2.3) (see [4, [10] and the references cited therein). Collocation and quadrature methods for solving numerically integral equations such as (2.3) are presented in 2. In that paper the authors develop widely the necessary theory for establishing the convergence results in weighted Sobolev spaces. In a subsequent paper [3], the solution by means of a polynomial collocation method is proposed and for this case uniform convergence results are thoroughly derived. Following the technique of [2, 3], we derive the collocation and quadrature methods to solve (1.1) with optimal convergence rates.

In the following we summarize some results concerning the properties of the hypersingular integral equation (2.3).

Lemma 2.2 ([6, Theorem 1]; [2, Corollary 2.8]). The finite part integral operator $\hat{S}_{\varphi}$ is a continuous isomorphism between the spaces $L_{\varphi}^{2, s+1}$ and $L_{\varphi}^{2, s}$. Moreover, for $u \in L_{\varphi}^{2, s+1}$,

$$
\hat{S}_{\varphi} u=\sum_{j=0}^{\infty}(j+1)\left\langle u, U_{j}\right\rangle_{\varphi} U_{j}
$$

where the series converges in the sense of $L_{\varphi}^{2, s}$.

Lemma 2.3 ([3, Proposition 2.3]). The inverse operator $\hat{S}_{\varphi}^{-1}: L_{\varphi}^{2, s} \rightarrow L_{\varphi}^{2, s+1}$ of the hypersingular integral operator $\hat{S}_{\varphi}$ can be written in the form

$$
\hat{S}_{\varphi}^{-1}:=S_{\sigma} W_{\sigma} S_{\varphi}
$$

where the continuous operators

$$
S_{\varphi}: L_{\varphi}^{2, s} \rightarrow L_{\sigma}^{2, s}, \quad W_{\sigma}: L_{\sigma}^{2, s} \rightarrow L_{\sigma}^{2, s+1}, \quad S_{\sigma}: L_{\sigma}^{2, s+1} \rightarrow L_{\varphi}^{2, s+1}
$$

are defined by

$$
\begin{gathered}
\left(S_{\varphi} u\right)(t)=\frac{1}{\pi} \int_{-1}^{1} \frac{u(x)}{x-t} \varphi(x) d x, \\
\left(W_{\sigma} u\right)(t)=\frac{1}{\pi} \int_{-1}^{1} \log |t-x| u(x) \sigma(x) d x, \\
\left(S_{\sigma} u\right)(t)=\frac{1}{\pi} \int_{-1}^{1} \frac{u(x)}{x-t} \sigma(x) d x,
\end{gathered}
$$

respectively. Moreover,

$$
\left(\hat{S}_{\varphi}^{-1}\right) U_{j}=\left(S_{\sigma} W_{\sigma} S_{\varphi}\right) U_{j}=\frac{U_{j}}{j+1}, \quad j=0,1,2, \ldots .
$$


The following lemma was proved for the first time by P. Junghanns in [8], in a different context. Here, we report a different proof, only for the clarity of the reader.

LEMma 2.4. Let $r \geq 0$ be an integer and $\gamma=g \varphi$. If $\left|\gamma^{(k)}\right| \varphi^{k} \leq$ const, $k=0,1, \ldots, r$, then $A_{\varphi} u \in L_{\varphi}^{2, r}$ when $u \in L_{\varphi}^{2, r}$.

Proof. Let $u \in L_{\varphi}^{2, r}$. Since $u \in L_{\varphi}^{2, r} \subseteq L_{\varphi}^{2}$, and $r$ is an integer, we have

$$
\int_{-1}^{1}\left[D^{k} u(x)\right]^{2}(1-x)^{k+1 / 2}(1+x)^{k+1 / 2} d x<\infty, \quad k=1,2, \ldots, r .
$$

Moreover $\gamma u \in L_{\varphi}^{2}, \gamma$ being bounded and $u \in L_{\varphi}^{2}$. Thus, to prove that $\gamma u \in L_{\varphi}^{2, r}$ it is sufficient to prove that

$$
\int_{-1}^{1}\left[D^{k}(\gamma(x) u(x))\right]^{2}(1-x)^{k+1 / 2}(1+x)^{k+1 / 2} d x<\infty, \quad k=1,2, \ldots, r
$$

(see also [1]). Now, by applying the Cauchy-Schwarz inequality,

$$
\begin{aligned}
& \int_{-1}^{1}\left[D^{k}(\gamma(x) u(x))\right]^{2}(1-x)^{k+1 / 2}(1+x)^{k+1 / 2} d x \\
& =\int_{-1}^{1}\left[\sum_{j=0}^{k}\left(\begin{array}{l}
k \\
j
\end{array}\right) D^{k-j} \gamma(x) D^{j} u(x)\right]^{2}(1-x)^{k+1 / 2}(1+x)^{k+1 / 2} d x \\
& =\int_{-1}^{1} \sum_{j=0}^{k}\left(\begin{array}{l}
k \\
j
\end{array}\right)^{2}\left[D^{k-j} \gamma(x)\right]^{2}\left[D^{j} u(x)\right]^{2}\left(1-x^{2}\right)^{k+1 / 2} d x \\
& +2 \int_{-1}^{1} \sum_{j=0}^{k-1}\left(\begin{array}{l}
k \\
j
\end{array}\right) D^{k-j} \gamma(x) D^{j} u(x) \sum_{i=j+1}^{k}\left(\begin{array}{c}
k \\
i
\end{array}\right) D^{k-i} \gamma(x) D^{i} u(x)\left(1-x^{2}\right)^{k+1 / 2} d x \\
& =\sum_{j=0}^{k}\left(\begin{array}{l}
k \\
j
\end{array}\right)^{2} \int_{-1}^{1}\left[D^{j} u(x)\right]^{2}\left(1-x^{2}\right)^{j+1 / 2}\left[D^{k-j} \gamma(x) \varphi^{k-j}\right]^{2} d x \\
& +2 \sum_{j=0}^{k-1} \sum_{i=j+1}^{k}\left(\begin{array}{l}
k \\
j
\end{array}\right)\left(\begin{array}{l}
k \\
i
\end{array}\right) \int_{-1}^{1} D^{k-j} \gamma(x) D^{k-i} \gamma(x) D^{j} u(x) D^{i} u(x)\left(1-x^{2}\right)^{k+1 / 2} d x \\
& \leq \operatorname{const} \sum_{j=0}^{k} \int_{-1}^{1}\left[D^{j} u(x)\right]^{2}\left(1-x^{2}\right)^{j+1 / 2} d x \\
& +2 \sum_{j=0}^{k-1} \sum_{i=j+1}^{k}\left(\begin{array}{l}
k \\
j
\end{array}\right)\left(\begin{array}{l}
k \\
i
\end{array}\right) \int_{-1}^{1}\left[D^{k-j} \gamma(x)\right]^{2}\left[D^{j} u(x)\right]^{2}\left(1-x^{2}\right)^{k+1 / 2} d x \\
& \times \int_{-1}^{1}\left[D^{k-i} \gamma(x)\right]^{2}\left[D^{i} u(x)\right]^{2}\left(1-x^{2}\right)^{k+1 / 2} d x<\infty,
\end{aligned}
$$

where we have used the assumptions on $u$ and $\gamma$. This proves the lemma.

LEMma 2.5. Let $s \geq 0$ and $\gamma=g \varphi$. If $\left|\gamma^{(k)}\right| \varphi^{k} \leq$ const, $k=0,1, \ldots,[s+1],[s+1]$ being the integer such that $s \leq[s+1] \leq s+1$, then the operator $A_{\varphi}: L_{\varphi}^{2, s+1} \rightarrow L_{\varphi}^{2, s}$ is compact. 
Proof. Since $[s+1] \leq s+1$ implies $L_{\varphi}^{2,[s+1]} \supseteq L_{\varphi}^{2, s+1}$, it follows that $u \in L_{\varphi}^{2,[s+1]}$. Thus, in view of the previous lemma and recalling that $L_{\varphi}^{2, r+\varepsilon}, r \geq 0, \varepsilon>0$, is compact imbedded in $L_{\varphi}^{2, r}$ (cf. [1]), the assertion follows.

Lemma 2.6. Let $k(\cdot, x) \in L_{\varphi}^{2, s+1+\varepsilon}, \varepsilon>0$ uniformly with respect to $x \in[-1,1]$, and $k_{x}(\cdot, x) \in L_{\varphi}^{2}$. Then the operator $\hat{K}_{\varphi}: L_{\varphi}^{2} \rightarrow L_{\varphi}^{2, s}$ is compact.

Proof. The assumptions assure that $k_{x}(\cdot, x) \in L_{\varphi}^{2, s+\varepsilon}$ uniformly with respect to $x \in$ $[-1,1]$. Thus, the assertion follows by applying Lemma 4.2 in [1].

We remark that the previous lemma remains true for other perturbation kernels $k$ (see [2]).

TheOREm 2.7. Assume that the hypotheses of Lemmas 2.5 and 2.6 are fulfilled. If $\operatorname{Ker}\left(A_{\varphi}+\hat{S}_{\varphi}+K_{\varphi}\right)=\{0\}$ in $L_{\varphi}^{2}$, then the operator $A_{\varphi}+\hat{S}_{\varphi}+\hat{K}_{\varphi}$ is bounded having an inverse.

Proof. The assertion follows by Lemmas 2.2, 2.5, 2.6.

3. Numerical algorithm. Finite and boundary element methods are two of the most frequently used numerical approaches for solving crack problems in fracture mechanics. An alternative approach is the integral equation method, which gives significant simplifications and advantages (e.g., it reduces a partial differential equation (PDE) in two dimensions to a one-dimensional singular integral equation (1D SIE)) and, in general, is more accurate than the aforementioned methods. This becomes obvious when it is possible to prove stability and convergence of a suitable numerical method to solve the 1D SIE. The convergence analysis of the numerical methods (e.g., finite elements, spectral method) to solve the same problems formulated by PDE accompanied by proper boundary conditions requires different arguments. We solve (1.3) numerically, proving stability and optimal convergence rates of the proposed procedure.

We investigate equation (2.3) in the pair of spaces $\left(L_{\varphi}^{2, s+1}, L_{\varphi}^{2, s}\right)$. Let $x_{n, j}^{\varphi}$ be the zeros of $U_{n}$ and denote by $\mathcal{L}_{n}^{\varphi}$ the Lagrange interpolation operator

$$
\left(\mathcal{L}_{n}^{\varphi} \delta\right)(x)=\sum_{j=1}^{n} \delta\left(x_{n, j}^{\varphi}\right) \ell_{n, j}^{\varphi}(x), \quad \ell_{n, j}^{\varphi}(x)=\prod_{i=1, i \neq j}^{n} \frac{x-x_{n, i}^{\varphi}}{x_{n, j}^{\varphi}-x_{n, i}^{\varphi}}, \quad j=1,2, \ldots, n .
$$

The collocation method consists in looking for an approximate solution $u_{n} \in \Pi_{n-1}$ of (2.3) by solving the equation

$$
\mathcal{L}_{n}^{\varphi}\left(A_{\varphi}+\hat{S}_{\varphi}+\hat{K}_{\varphi}\right) u_{n}=\mathcal{L}_{n}^{\varphi} h,
$$

$\Pi_{n-1}$ being the set of all polynomials of degree at most $n-1$. In view of Lemma 2.1 and $\hat{S}_{\varphi} u=\sum_{j=0}^{\infty}(j+1)\left\langle u, U_{j}\right\rangle_{\varphi} U_{j}$, this equation is equivalent to

$$
\left(I+\hat{S}_{\varphi}^{-1} L_{n}^{\varphi}\left(A_{\varphi}+\hat{K}_{\varphi}\right)\right) u_{n}=\hat{S}_{\varphi}^{-1} L_{n}^{\varphi} h .
$$

The following theorems prove the stability and the convergence of the proposed method. 
Theorem 3.1. Assume that the hypotheses of Lemmas 2.5 and 2.6 are fulfilled. If $\operatorname{Ker}\left(A_{\varphi}+\hat{S}_{\varphi}+\hat{K}_{\varphi}\right)=\{0\}$ in $L_{\varphi}^{2}$, then for sufficiently large $n$ the operator $I+\hat{S}_{\varphi}^{-1} \mathcal{L}_{n}^{\varphi}\left(A_{\varphi}+\hat{K}_{\varphi}\right)$ has a bounded inverse in $L_{\varphi}^{2, s+1}$.

Proof. In view of Lemmas 2.5, 2.6 the operator

$$
A_{\varphi}+\hat{K}_{\varphi}: L_{\varphi}^{2, s+1} \rightarrow L_{\varphi}^{2,[s+1]-\varepsilon},
$$

is compact. Further

$$
\left\|\hat{S}_{\varphi}^{-1}\left(\mathcal{L}_{n}^{\varphi}-I\right)\left(A_{\varphi}+\hat{K}_{\varphi}\right)\right\|_{L_{\varphi}^{2, s+1} \rightarrow L_{\varphi}^{2, s+1}} \leq \mathrm{const}\left\|\left(\mathcal{L}_{n}^{\varphi}-I\right)\left(A_{\varphi}+\hat{K}_{\varphi}\right)\right\|_{L_{\varphi}^{2, s+1} \rightarrow L_{\varphi}^{2,[s+1]-\varepsilon}},
$$

where we have used Lemma 2.2. On the other hand,

$$
\lim _{n \rightarrow \infty}\left\|\delta-\mathcal{L}_{n}^{\varphi} \delta\right\|_{\varphi,[s+1]-\varepsilon}=0, \quad \delta \in L_{\varphi}^{2,[s+1]-\varepsilon},
$$

since $[s+1]-\varepsilon>1 / 2$ with suitable $\varepsilon>0$ (see Theorem 3.4 in [1]). Thus

$$
\left\|\hat{S}_{\varphi}^{-1}\left(\mathcal{L}_{n}^{\varphi}-I\right)\left(A_{\varphi}+\hat{K}_{\varphi}\right)\right\|_{L_{\varphi}^{2, s+1} \rightarrow L_{\varphi}^{2, s+1}} \rightarrow 0
$$

and, consequently,

$$
\left\|\left(I+\hat{S}_{\varphi}^{-1} \mathcal{L}_{n}^{\varphi}\left(A_{\varphi}+\hat{K}_{\varphi}\right)\right)^{-1}\right\|_{L_{\varphi}^{2, s+1} \rightarrow L_{\varphi}^{2, s+1}} \leq \text { const. }
$$

This proves the theorem.

Theorem 3.2. Assume that the hypotheses of Lemmas 2.5 and 2.6 are fulfilled. If the constant $C$ in (1.2) and the given functions $g, f, k$ are such that $h \in L_{\varphi}^{2, r}$, and $\left(A_{\varphi}+\hat{K}_{\varphi}\right) u \in L_{\varphi}^{2, r}$, with $r \geq s, r>1 / 2$, then for sufficiently large $n$,

$$
\left\|u-u_{n}\right\|_{\varphi, s+1} \leq \frac{\text { const }}{n^{r-s}}\left\{\|h\|_{\varphi, r}+\left\|\left(A_{\varphi}+\hat{K}_{\varphi}\right) u\right\|_{\varphi, r}\right\} .
$$

Proof. Equation (2.3) can be rewritten as follows:

$$
\left[I+\hat{S}_{\varphi}^{-1} \mathcal{L}_{n}^{\varphi}\left(A_{\varphi}+\hat{K}_{\varphi}\right)\right] u+\hat{S}_{\varphi}^{-1}\left(A_{\varphi}+\hat{K}_{\varphi}\right) u-\hat{S}_{\varphi}^{-1} \mathcal{L}_{n}^{\varphi}\left(A_{\varphi}+\hat{K}_{\varphi}\right) u=\hat{S}_{\varphi}^{-1} h .
$$

By (2.3) and (3.2) we deduce

$$
\begin{aligned}
{\left[I+\hat{S}_{\varphi}^{-1} \mathcal{L}_{n}^{\varphi}\left(A_{\varphi}+\hat{K}_{\varphi}\right)\right]\left(u-u_{n}\right)=} & -\hat{S}_{\varphi}^{-1}\left(A_{\varphi}+\hat{K}_{\varphi}\right) u \\
& +\hat{S}_{\varphi}^{-1} \mathcal{L}_{n}^{\varphi}\left(A_{\varphi}+\hat{K}_{\varphi}\right) u+\hat{S}_{\varphi}^{-1} h-\hat{S}_{\varphi}^{-1} \mathcal{L}_{n}^{\varphi} h,
\end{aligned}
$$

which implies that

$$
\begin{aligned}
{\left[I+\hat{S}_{\varphi}^{-1} \mathcal{L}_{n}^{\varphi}\left(A_{\varphi}+\hat{K}_{\varphi}\right)\right]\left(u-u_{n}\right)=} & \hat{S}_{\varphi}^{-1}\left\{\left(h-\mathcal{L}_{n}^{\varphi} h\right)\right. \\
& \left.+\left[\mathcal{L}_{n}^{\varphi}\left(A_{\varphi}+\hat{K}_{\varphi}\right)-\left(A_{\varphi}+\hat{K}_{\varphi}\right)\right] u\right\} .
\end{aligned}
$$

Applying Theorem 3.1 together with Lemma 2.2 we obtain

$$
\left\|u-u_{n}\right\|_{\varphi, s+1} \leq\left\|h-\mathcal{L}_{n}^{\varphi} h\right\|_{\varphi, s}+\left\|\left(I-\mathcal{L}_{n}^{\varphi}\right)\left(A_{\varphi}+\hat{K}_{\varphi}\right) u\right\|_{\varphi, s} .
$$


Moreover, in view of Theorem 3.4 in [1, we have

$$
\begin{aligned}
\left\|h-\mathcal{L}_{n}^{\varphi} h\right\|_{\varphi, s} & \leq \frac{\text { const }}{n^{r-s}}\|h\|_{\varphi, r}, \\
\left\|\left(I-\mathcal{L}_{n}^{\varphi}\right)\left(A_{\varphi}+\hat{K}_{\varphi}\right) u\right\|_{\varphi, s} & \leq \frac{\text { const }}{n^{r-s}}\left\|\left(A_{\varphi}+\hat{K}_{\varphi}\right) u\right\|_{\varphi, r},
\end{aligned}
$$

since $h \in L_{\varphi}^{2, r}$ and $\left(A_{\varphi}+\hat{K}_{\varphi}\right) u \in L_{\varphi}^{2, r}$, with $r \geq s$ and $r>1 / 2$. Comparing (3.3)-(3.5), the theorem follows.

With the help of $Q_{n}^{\varphi}$ we will denote the application of the Gaussian rule with respect to the weight $\varphi$, which means that

$$
\int_{-1}^{1} \delta(t) \varphi(t) d t \approx Q_{n}^{\varphi}(\delta)=\sum_{j=1}^{n} \lambda_{n, j}^{\varphi} \delta\left(x_{n, j}^{\varphi}\right),
$$

with

$$
\lambda_{n, j}^{\varphi}=\int_{-1}^{1} \ell_{n, j}^{\varphi}(x) \varphi(x) d x, \quad j=1,2, \ldots, n .
$$

Now, we can approximate the operator $\hat{K}_{\varphi}$ by

$$
\left(\hat{K}_{n}^{\varphi} u\right)(t)=\frac{1}{\pi} \sum_{j=1}^{n} \lambda_{n, j}^{\varphi} k_{x}\left(t, x_{n, j}^{\varphi}\right) u\left(x_{n, j}^{\varphi}\right) .
$$

The quadrature or discrete collocation method consists in solving the equation to

$$
\left(I+\hat{S}_{\varphi}^{-1} L_{n}^{\varphi}\left(A_{\varphi}+\hat{K}_{n}^{\varphi}\right)\right) u_{n}^{*}=\hat{S}_{\varphi}^{-1} L_{n}^{\varphi} h .
$$

Lemma 3.3. Assume $k(t, \cdot) \in L_{\varphi}^{2, s+1}$, for some $s>1 / 2$ uniformly with respect to $t \in[-1,1]$, and $k_{x}(t, \cdot) \in L_{\varphi}^{2}$. Then, for $0 \leq r \leq s$ and $u \in L_{\varphi}^{2}$,

$$
\left\|\mathcal{L}_{n}^{\varphi}\left(\hat{K}_{n}^{\varphi}-\hat{K}_{\varphi}\right) u\right\|_{\varphi, r+1} \leq \text { const } n^{r-s}\|u\|_{\varphi}
$$

Proof. The assumptions assure that $k_{x}(t, \cdot) \in L_{\varphi}^{2, s}$ uniformly with respect to $t \in[-1,1]$ (see [1]). Since, for a polynomial $p_{n}$ of degree less than $n,\left\|p_{n}\right\|_{\varphi, r+1} \leq n^{r+1}\left\|p_{n}\right\|_{\varphi}$, we are able to estimate, with the help of the Schwarz inequality,

$$
\begin{aligned}
\| \mathcal{L}_{m}^{\varphi}\left(\hat{K}_{n}^{\varphi}\right. & \left.-\hat{K}_{\varphi}\right) u\left\|_{\varphi, r+1}^{2} \leq n^{2 r+2}\right\| \mathcal{L}_{n}^{\varphi}\left(\hat{K}_{n}^{\varphi}-\hat{K}_{\varphi}\right) u \|_{\varphi}^{2} \\
& =\frac{n^{2 r+2}}{\pi} \sum_{j=1}^{n} \lambda_{n, j}^{\varphi}\left\{\int_{-1}^{1} u(x)\left[\left(\mathcal{L}_{n}^{\varphi} k_{x}\left(x_{n, j}^{\varphi}, \cdot\right)\right)(x)-k_{x}\left(x_{n, j}^{\varphi}, x\right)\right] \varphi(x) d x\right\}^{2} \\
& \leq \frac{n^{2 r+2}}{\pi}\|u\|_{\varphi}^{2} \sum_{j=1}^{n} \lambda_{n, j}^{\varphi}\left\|\left(\mathcal{L}_{n}^{\varphi} k_{x}\left(x_{n, j}^{\varphi}, \cdot\right)\right)-k_{x}\left(x_{n, j}^{\varphi}, \cdot\right)\right\|_{\varphi}^{2} \\
& \leq \mathrm{const} n^{2 r-2 s}\|u\|_{\varphi}^{2} \sum_{j=1}^{n} \lambda_{n, j}^{\varphi}\left\|k_{x}\left(x_{n, j}^{\varphi}, \cdot\right)\right\|_{\varphi, s+1}^{2} \\
& \leq \mathrm{const} n^{2 r-2 s}\|u\|_{\varphi}^{2},
\end{aligned}
$$

where we have used that for all $\delta \in L_{\varphi}^{2, s}$,

$$
\left\|\delta-\mathcal{L}_{n}^{\varphi}(\delta)\right\|_{\varphi} \leq \text { const } n^{-s}\|\delta\|_{\varphi, s} \quad \text { if } s>\frac{1}{2}
$$


(see [1]).

The next theorem proves the convergence and the stability of the discrete collocation method.

Theorem 3.4. Assume that the hypotheses of Theorems 3.1 and 3.2 are fulfilled. If $k(t, \cdot) \in L_{\varphi}^{2, s+1}$, for some $s>1 / 2$ uniformly with respect to $t \in[-1,1]$, and $k_{x}(t, \cdot) \in L_{\varphi}^{2}$, then, for sufficiently large $n$, equation (3.6) is uniquely solvable, and the solution $u_{n}^{*}$ converges in the norm of the space $L_{\varphi}^{2, r+1}, 0 \leq r<s$, to the unique solution $u$ of (2.3), where

$$
\left\|u_{n}^{*}-u\right\|_{\varphi, r+1} \leq \text { const } n^{r-s}\|u\|_{\varphi, s+1} .
$$

Proof. Taking into account Lemma 3.3, the proof goes along the same lines as the proof of Theorem 3.8 in [2].

Now, we come back to the original unknown. As we can deduce from relation (2.4), we can write

$$
\bar{v}(t)=\varphi(t) \frac{d}{d t}[\varphi(t) u(t)]+\frac{C}{2} \varphi(t) .
$$

Moreover, we consider the approximate solution $u_{n}(t)=\sum_{k=0}^{n-1} a_{k} U_{k}(t)$ of the hypersingular integral equation (2.3), where the coefficients $a_{k}$ are obtained by solving, as usual, the linear system (3.1) for the collocation method and the linear system (3.6) in the discrete collocation method. In view of relation (2.1) of Theorem 2.1, we obtain an approximate solution

$$
\bar{v}_{n}(t)=-\sum_{k=0}^{n-1}(k+1) a_{k} T_{k+1}(t)+\frac{C}{2} \varphi(t),
$$

of the original equation (1.3). Furthermore, it is easy to see from relation (2.2) of Theorem 2.1 that

$$
\left\|\bar{v}-\bar{v}_{n}\right\|_{\tilde{H}_{\sigma}^{2, s}} \leq \mathrm{const}\left\|u-u_{n}\right\|_{\varphi, s+1} .
$$

4. Numerical considerations. In this section we state some numerical results obtained using the algorithm described in Section 3. All computations reported in this section were carried out in double precision. We observe that the numerical results are in accordance with the theoretical ones. Furthermore, we point out some computational aspects regarding the numerical resolution of the linear system (3.1).

EXAMPLE 4.1. We solve the integral equation (1.1) with $g(t)=1 / \sqrt{1-t^{2}}, k(t, x)=$ $t^{2}|t| x+\frac{1}{8}\left[x^{2}|x| \sqrt{1-x^{2}}-2|x| \sqrt{1-x^{2}}+2 \arcsin |x|\right]$ and $f(t)=\frac{t|t|}{4}(4-t)+\frac{4}{15} \pi-$ $\frac{t}{\pi}\left[\frac{2-3 t^{2}}{\sqrt{1-t^{2}}} \log \frac{1+\sqrt{1-t^{2}}}{1-\sqrt{1-t^{2}}}-6\right]$. Assuming that $\int_{-1}^{1} v(x) d x=0$, the solution is given by $v(t)=2|t|$. We remark that the solution $u(t)=t|t|$ of $(2.3)$ is such that $u \in L_{\varphi}^{2,2.5-\epsilon}$ for all $\epsilon>0$. Moreover the convergence rate given by Theorem 3.2 for $s=0$ is $\left\|u-u_{n}\right\|_{\varphi, 1}=$ $O\left(n^{\epsilon-1.5}\right)$, for $\epsilon$ arbitrarily small. Thus, in view of (3.8), we deduce $\left\|\bar{v}-\bar{v}_{n}\right\|_{\sigma} \leq O\left(n^{\epsilon-1.5}\right)$. This convergence rate is confirmed by the numerical results given in Table 1 . 
TABLE 1

\begin{tabular}{|r|r|}
\hline$n$ & $\left\|\bar{v}-\bar{v}_{n}\right\|_{\sigma}$ \\
\hline \hline 8 & $3.53 \mathrm{D}-02$ \\
16 & $1.43 \mathrm{D}-02$ \\
32 & $5.43 \mathrm{D}-03$ \\
64 & $2.01 \mathrm{D}-03$ \\
128 & $7.21 \mathrm{D}-04$ \\
256 & $2.58 \mathrm{D}-04$ \\
512 & $9.15 \mathrm{D}-05$ \\
\hline
\end{tabular}

TABLE 2

\begin{tabular}{|r|r|}
\hline$n$ & $\left\|\bar{v}_{4096}-\bar{v}_{n}\right\|_{\sigma}$ \\
\hline \hline 8 & $6.35 \mathrm{D}-02$ \\
16 & $1.79 \mathrm{D}-02$ \\
32 & $4.99 \mathrm{D}-03$ \\
64 & $1.37 \mathrm{D}-03$ \\
128 & $3.70 \mathrm{D}-04$ \\
256 & $9.87 \mathrm{D}-05$ \\
512 & $2.59 \mathrm{D}-05$ \\
\hline
\end{tabular}

ExAmple 4.2. In this example we solve the integral equation (1.1) with $g(t)=$ $1 / \sqrt{1-t^{2}}, k(t, x)=0, f(t)=\left(t^{2}-3\right)|t|-\log \frac{1-t}{1+t}+\pi \sqrt{\frac{1+t}{1-t}}$ and assuming $\int_{-1}^{1} v(x) d x=2 \pi$. In this case the solution $v$ is unknown. For this reason we compare the approximate solution $\bar{v}_{n}$ with $\bar{v}_{4096}$. Since $h=\left(t^{2}-3\right)|t|$, we deduce that $h \in L_{\varphi}^{2,1.5-\epsilon}$, and Theorem 3.2 shows the following convergence rate: $\left\|u-u_{n}\right\|_{\varphi, 1}=O\left(n^{\epsilon-1.5}\right)$, for $s=0$ and with $\epsilon$ arbitrarily small. Thus, in view of (3.8), we deduce $\left\|\bar{v}-\bar{v}_{n}\right\|_{\sigma} \leq O\left(n^{\epsilon-1.5}\right)$. Also in this case the numerical results of Table 2 confirm the theoretical ones.

Finally, we remark that if the unknown function $\bar{v}$ is even, as happens in some particular contact problems, in view of (2.4) it follows that $u$ is odd. For this reason, there is a reduction to zero of half of the coefficients of the Chebyshev expansion $u_{n}(t)=\sum_{k=0}^{n-1} a_{k} U_{k}(t)$.

Acknowledgment. The authors are grateful to the referee for his contribution in improving the paper.

\section{REFERENCES}

[1] D. Berthold, W. Hoppe, B. Silbermann, A fast algorithm for solving the generalized airfoil equation, J. Comput. Appl. Math. 43 (1992), 185-219. MR.1193301 (93k:65106)

[2] M. R. Capobianco, G. Criscuolo, P. Junghanns, A fast algorithm for Prandtl's integro-differential equation, J. Comput. Appl. Math. 77 (1997), 103-128. MR.1440006 (98a:65186)

[3] M. R. Capobianco, G. Criscuolo, P. Junghanns, U. Luther, Uniform convergence of the collocation method for Prandtl's integro-differential equation, ANZIAM J. 42 (2000), 151-168. MR1783378 (2001i:65138) 
[4] R. Chapko, R. Kress, L. Monch, The numerical solution of a hypersingular integral equation for elastic scattering from a planar crack, IMA J. Numer. Anal. 20 (2000), 601-619. MR1795299 (2002c:45012)

[5] J. A. Cuminato, A. D. Fitt, S. McKee, A review of linear and nonlinear Cauchy singular integral and integro-differential equations arising in mechanics, J. Integral Equations Appl. 19 (2007), 163-207. MR2355008 (2009b:45009)

[6] V. J. Ervin, E. P. Stephan, Collocation with Chebyshev polynomials for a hypersingular integral equation on an interval, J. Comput. Appl. Math. 43 (1992), 221-229. MR.1193302 (93j:65221)

[7] G. M. L. Gladwell, Contact Problems in the Classical Theory of Elasticity, Sijthof, Noordhoff, The Netherlands, 1980. MR661763 (84m:73094)

[8] P. Junghanns, Product integration for the generalized airfoil equation, in Eberhard Schock, Ed., Beiträge zur angewandten Analysis und Informatik (Shaker, Aachen, 1994), 171-188. MR1287178 (95d:45005)

[9] A. I. Kalandija, Mathematichal Methods of Two-Dimensional Elasticty, Mir Publishers, Moscow, 1975. MR 0400846 (53:4676)

[10] A. C. Kaya, F. Erdogan, On the solution of integral equations with strongly singular kernels, Quart. Appl. Math., 45 (1987), 105-122. MR0885173(88e:45012) 